\title{
La scuola ai tempi della pandemia. Intervista ad Andrea Canevaro
}

\author{
di Nadia Olivieri
}

Ott 7, $2020|\underline{\text { In evidenza, }} \underline{\text { Pensare la didattica }}| \underline{0} \mid$

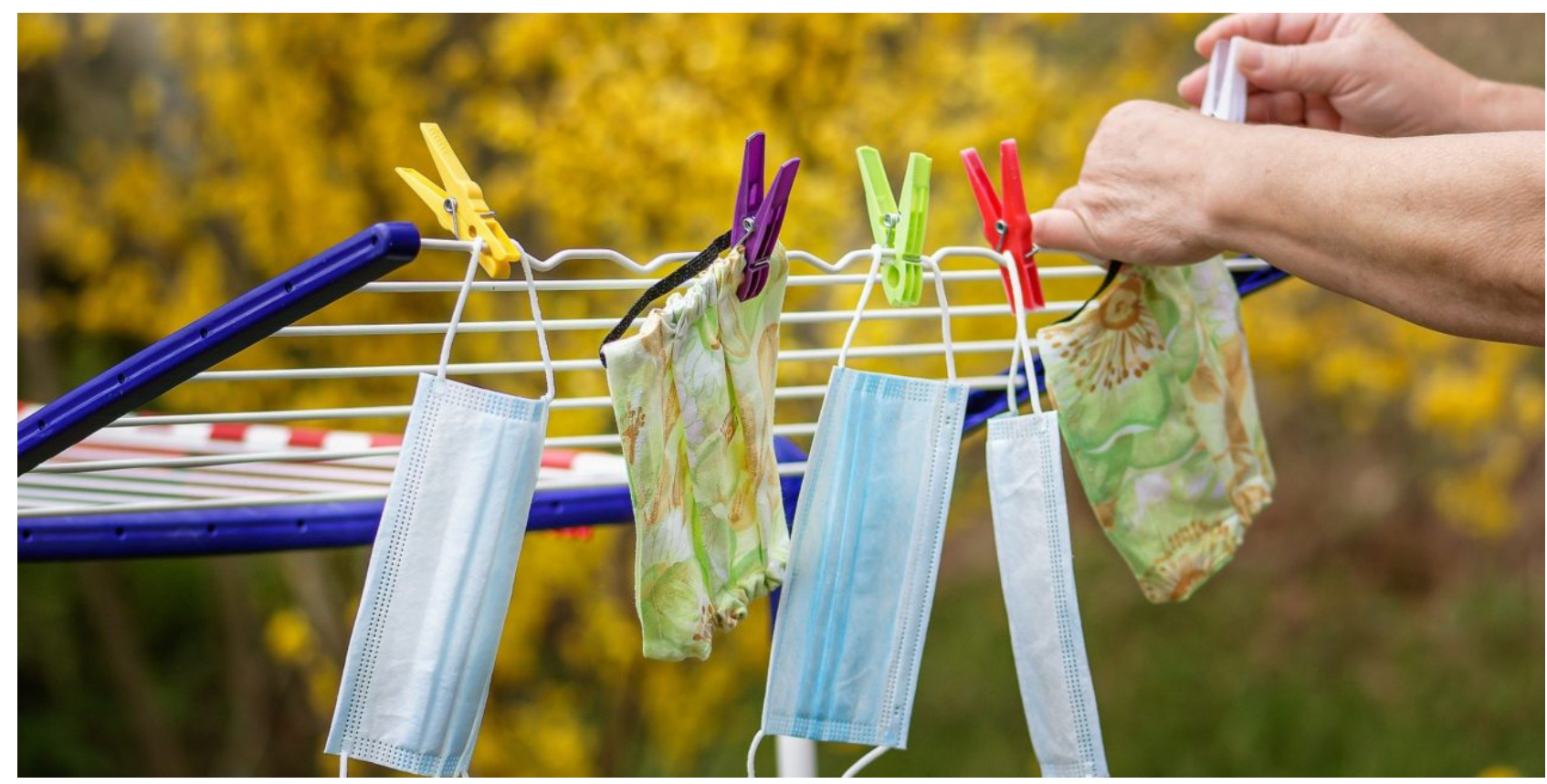

dida

\begin{abstract}
Nei mesi scorsi la pandemia da Covid-19 ha investito la scuola in modo inatteso e, a fronte dell'emergenza sanitaria, si è messa in atto una "Didattica dell'emergenza". La totale immersione nel lavoro che ha caratterizzato la vita degli insegnanti nei mesi di lockdown ha spesso impedito una riflessione su quanto è successo, sul tipo di didattica impartita e sulla relazione educativa che questa ha imposto.

Ora è tempo di "ri-pensare la didattica", sia quella agita sia, e ancor più, quella auspicabile. La redazione ha perciò deciso di chiedere ad Andrea Canevaro, pedagogista e professore emerito dell'Università degli studi di Bologna che si è a lungo occupato di inclusione sociale, quali sono le sue considerazioni su quanto accaduto e quali consigli si sente di dare agli insegnanti ad anno scolastico appena iniziato.
\end{abstract}

Partiamo dal termine emergenza. Mentre le sto ponendo questa domanda è ancora in vigore, in Italia, lo stato di emergenza proclamato dal governo per fronteggiare la pandemia. L'emergenza ha letteralmente travolto il mondo della scuola; la necessità del distanziamento fisico fra le persone ha impedito la conclusione in presenza dell'anno scolastico 2019-2020. Improvvisamente tutto è cambiato. Quali considerazioni si possono fare, ora, su tutto questo?

Questa emergenza mi ha fatto riandare col pensiero ad un'altra emergenza: quella degli inizi degli anni Novanta durante la guerra nella ex Jugoslavia. Tuzla è una città della Bosnia Erzegovina e nel corso del conflitto le sue scuole sono chiuse. Un insegnante di matematica, volendo rimanere in 
qualche modo in contatto con gli allievi, organizza una piccola radio locale che aveva un programma radiofonico di matematica. I ragazzi e le ragazze sentivano la voce del loro insegnante e così veniva mantenuto un legame di appartenenza. Gli accordi di Dyton[1], nel 1995, determinarono la fine alla guerra e nacque la Federazione Bosnia Erzegovina. Le scuole riaprirono. Quell'insegnante assunse un ruolo politico nel governo del Cantone di Tuzla. Poteva, per risparmiare, proporre la radio come modo di fare lezione di matematica per tanti, risparmiando sugli stipendi di un certo numero di insegnanti. Non volle. L'emergenza era finita. Non sappiamo cosa accadde alla piccola emittente. Ci piace immaginare che non abbia smesso di trasmettere. Potrebbe aver continuato a funzionare grazie all'impegno delle ragazze e dei ragazzi, capaci di mandare avanti un programma radiofonico impostato sulla matematica.

Più o meno negli stessi anni del dopoguerra bosniaco, in Romagna si presenta un diverso tipo di emergenza. In un istituto scolastico tecnico superiore un allievo è affetto da una grave malattia degenerativa che lo tiene a casa, a letto. Nasce il Progetto Maometto. Come dice il proverbio: «Se la montagna non va a Maometto...» sarà la scuola ad andare a casa del ragazzo. Il gruppo che compone la sua classe, una mattina alla settimana, va a casa sua - dotata di un ingresso ampio, su cui si affaccia la camera del ragazzo - e, tenendo la porta spalancata e avvicinando il letto, riesce a ristabilire un possibile incontro per riferire al compagno ciò che hanno fatto e imparato a scuola. A fine anno scolastico, non solo il ragazzo era rimasto in contatto coi compagni, ma quel gruppo di ragazze e di ragazzi ebbe un rendimento scolastico eccellente.

Sono passati quasi trent'anni e adesso è arrivata la pandemia da Covid-19. Nuova emergenza e scuole chiuse. Bisogna inventarsi qualcosa. Ecco, la prima considerazione che mi viene da fare è che proprio in momenti come questi occorre assumersi il rischio di essere innovativi, di tentare soluzioni nuove; talvolta da un incastro, o un intreccio, di singole operosità si creano realtà feconde e produttive.

\section{Gli esempi fatti sono molto suggestivi, ma molto diversi fra loro. Nel caso di Tuzla, come ora con il Covid-19, la possibilità di raggiungere gli studenti dipendeva esclusivamente dalla tecnologia. Il dubbio che emerge è che la tecnologia condizioni enormemente la qualità della comunicazione o, addirittura, la comunicazione stessa.}

Il mediologo canadese Marshall Mc Luhan (1911-1980) ha forgiato, negli anni Sessanta del secolo scorso, l'espressione villaggio globale. Ed ha diffuso l'ipotesi che il medium potesse diventare il messaggio. Queste indicazioni ebbero una fortuna tale, da creare una volgarizzazione superficiale immediata, ed un'altrettanta immediata caduta nel vuoto della memoria. Forse però val la pena riprenderle e cercare di capire che il loro autore, al di là della moda, apriva prospettive di riflessione non di poco conto. Il messaggero - o il messaggio? - diventa, in alcuni casi, colui - o ciò - che fa irrompere nella nostra razionalità un elemento irrazionale. Può creare effetti dadaisti. Dovrebbe sorprenderci. E dovremmo preoccuparci se, anziché sorprenderci, reagissimo sulla stessa linea comunicativa. Una catena di reazioni frammentarie che non costituisce un senso prospettico.

Il medium, invece, non coincide con i messaggi. La radio che a Tuzla faceva matematica era, tecnologicamente, identica a quella che, in quegli stessi anni, costituiva un potente mezzo per la realizzazione del genocidio ruandese[2]. Tra medium e messaggio ci siamo noi, con le nostre intenzioni, i nostri progetti, le nostre parole. Raccontare ad altri significa riconoscere negli altri dei valori. Levinas diceva che «Riconoscere altri significa [...] raggiungerl[i] attraverso il mondo delle cose possedute, ma, simultaneamente, instaurare con il dono la comunità e l'universalità. Il linguaggio è universale [...] perché è il passaggio dall'individuale al generale, perché offre cose 
mie ad altri»[3]. Con il linguaggio entra in scena un ecosistema formidabile. La parola ci permette di passare dal piano della realtà a quello dei simboli.

\section{Ci può fare un esempio concreto di questo passaggi?}

Pensiamo alla parola orizzonte. L'orizzonte è l'incontro della terra col cielo. E del cielo con la terra. È un'immagine. Chi non la vede in natura perché è cieco, l'ha nella mente. In buona parte è frutto delle parole. Orizzonte. Cielo. Terra. L'amico cieco pronuncia queste parole in maniera sensata. Queste parole hanno un senso anche senza poter vedere l'immagine in natura. Il prigioniero in una cella senza finestre ha in mente un orizzonte. Si allargherà quando uscirà di prigione; anche se dovesse uscire per andare ad abitare in una cantina.

Per gli esseri umani, un orizzonte ha un significato che va oltre l'immagine in natura. L'orizzonte di chi si è smarrito è ben diverso da quello del nomade che ha un punto di riferimento. Anche se i due fossero collocati nello stesso punto: lo stesso deserto avrebbe più di un orizzonte. L'orizzonte per Galileo era un invito ad andare oltre. Non così per altri. Per i suoi oppositori e per gli indifferenti. La stessa parola, secondo la mente che la ospitava, assumeva significati ben diversi. Rendere simbolico un fenomeno è tentare di superare le differenze, collocandolo "oltre". Il simbolo fa incontrare, per un progetto comune, diversità che possono essere lette con lo schema dell'aut-aut. Il simbolo fa scoprire l'et-et.

L'adesione a un simbolo permette di allargare l'orizzonte. Non necessariamente un simbolo religioso. Nel simbolo c'è il riconoscimento di un'appartenenza. È probabile che la parola appartenenza piaccia a chi legge oggi. Ma un simbolo può anche escludere, proponendo un'appartenenza esclusiva. La storia di cui facciamo parte, a cui apparteniamo, è fatta di esclusioni palesi o occulte. Non tutti apparteniamo alla storia. Per questo si dice la nostra storia non è la loro.

Ne dobbiamo dedurre che, anche e soprattutto con le parole, siamo messi di fronte alla responsabilità di scelte. La polisemia, la molteplicità di significati di una parola, ci mette di fronte $\mathrm{a}$ scelte, e di fronte a una scelta preliminare: scappare rintanandoci nella nostra ignoranza presuntuosa; o andare avanti con la curiosità che alimenta un dialogo accompagnato da riflessione.

In questo periodo, credo che sui media la parola appartenenza abbia cambiato connotazione rispetto alla chiusura identitaria con cui è stata spesso declinata negli ultimi tempi. Di fronte alla pandemia è stata sottolineata la comune appartenenza al genere umano, la condivisione di una condizione collettiva di pericolo davanti ad un rischio concreto. Sono impressioni corrette?

Sì, è così, ma solo in parte. Nelle nostre città vedo il rischio che il distanziamento sociale imposto dal coronavirus ci abitui ad avere scarsa attenzione nei confronti degli altri. Alexander Mitscherlich, alcuni anni fa, scriveva: «La città in cui si è vissuti per secoli era un biotopo [...] un luogo nel quale le più diverse forme di vita raggiungono un equilibrio ed in esso persistono»[4]. Il biotopo è un raro esempio, nelle discipline naturali, di coesistenza non esclusivamente parassitaria. Esso è composto di elementi che sono funzionamenti e non funzioni. Ma la città dei nostri giorni si è trasformata in un insieme di funzioni, ciascuna con un suo compito esclusivo: il quartiere degli affari, quello dormitorio, quello dello svago, e magari quello a luci rosse. Le monofunzioni non possono che essere fonti di discordia: moltiplicano situazioni disgregate, in cui il soggetto non riesce a vivere in 
modo omogeneo alla funzione in cui è collocato. Ad esempio: chi vive nel quartiere dormitorio e vorrebbe godersi la propria tranquillità, ha come vicino chi vive la casa anche per ricevere amici e sentire musica. Le monofunzioni non rispondono alle esigenze di una società complessa come la nostra. Il risultato è un aumento della micro-conflittualità e delle devianze. Per questo il nostro modo di vivere deve essere accompagnato da seduzioni di ogni tipo, vere e proprie droghe sociali (aumento di ogni tipo di dipendenze), che sovente vengono riassunte nel termine consumismo, che fa sembrare del tutto normale l'indebitamento permanente di ciascuno e di tutti. E l'indebitamento generalizzato comporta - va da sé - il sentimento di ciascuno e di tutti di essere non solo debitori ma anche creditori. E di risarcirsi, a volte, in maniera clamorosamente asociale. Ogni struttura complessa è imperfetta. Per nostra fortuna, ogni struttura complessa è però anche evolutiva.

Rada Ivekovic sostiene che una società, in periodi di particolare crisi che hanno la dinamica della guerra (aggiungiamo: anche le piccole guerre di "condominio intraprofessionale"), perde la sua coerenza epistemologica. Questo significa «che lo scarto tra la realtà e l'immagine di sé aumenta e che il loro legame può anche spezzarsi. Ci sarà allora una rottura della rappresentazione e, implicitamente, una sorta di scissione ermeneutica. Più la ferita è grande e più la società si fonda o piuttosto si rifonda - su una menzogna o su un ideale che poco ha a che vedere con la realtà. In tal caso, prima o poi, la non-verità verrà a galla e un nuovo paradigma, qualunque esso sia, verrà proposto»[5]. Rada Ivekovic è di Zagabria, ha studiato a Belgrado e a Delhi, e insegna a Paris VII. Ha preferito esiliarsi in Francia, e non vivere in una terra divisa in tante piccole patrie, nessuna delle quali poteva essere sentita come sua. Aveva la necessità di allargare lo spazio e abitare il mondo. Come allargare l'orizzonte e non rimanere prigionieri delle emergenze nelle nostre piccole patrie? Il nostro linguaggio, non imprigionato nei gerghi degli stereotipi, può servire per cercare di rispondere a questa domanda.

\section{Il racconto di questa particolarissima crisi ha quindi bisogno di un nuovo linguaggio?}

Abbiamo bisogno di interrogarci, di imparare a ragionare per "contesti" e di chiederci con quale linguaggio li abitiamo. Un contesto è diverso da una situazione. Può contenere elementi che vanno oltre i puri dati sensoriali. Un contesto ha un senso complessivo. È questo che dobbiamo cercare di restituire. Una insegnante di lingua e letteratura bosniaca nella scuola elettronica di Tuzla, Lejla Idrizovic, ha scritto, a tarda sera, all'inizio di aprile di quest'anno:

L'insoddisfazione è come una bestia, impotente quando nasce, terribile quando diventa più forte. Davanti a me c'è un compito molto difficile. Forse il più difficile. [...] lo paragono al testamento scritto da un vecchio nel momento in cui sente che è arrivato il tempo di separarsi e lasciare un segno.

Proprio ieri sono stata felice di tutto ciò che sono e di quello che sarò, ma oggi sono vittima di qualcosa di sconosciuto.

Nel frattempo, mi sveglio, [...] interrogandomi, chiamando me stessa, [e] ogni volta, l'ansia è la stessa.

La stessa paura e ansia, e la domanda: cosa porta il momento successivo? [...] siamo ancora impotenti.

La paura della fine, ma anche paura della vita, a cui anche i più coraggiosi non resistono. Mi sembra che, con ogni nuovo passo, tutto ciò mi porta più vicino a scomparire, e mi allontana dalla vita. Mi preoccupa che a volte non mi interessi, tranne per il fatto che sceglie lei quando e come venire. Se solo sapessi da quale parte verrà, se verrà [...].

Ho scritto una lettera, senza un indirizzo, solo il nome e il numero... "Covid-19":

«Presentati chi sei

mostra la tua faccia, se ne hai,

non essere un'ombra, senza testa 
che vaga a cercare corpi,

per appropriarsene, per diventare finale.

Lascia questo mio mondo,

e ritrovati nel tuo mondo,

quindi allontanati da me, da tutti miei

Voglio dirti dall'inizio!»

Sto bene quando creo, o mentre guardo "le mie" piccole / grandi "'persone"” (gli alunni) che creano. $[\ldots]$

La vita è bella, dopo tutto, solo bisogna sapere come leggerla. Per coincidenza, viene una musica dalla radio: "tutto è una bugia, tranne la morte».

Sì, davvero tutto ciò che viviamo, tutto ciò che creiamo, tutto ciò per cui lottiamo, tutto è niente. Mi chiedo: ma è niente la mia paura, che causa insoddisfazione e impotenza? Chi lo saprà? L'orologio sta ticchettando, i sogni colorati del passato stanno lentamente svanendo, e io, apro il mio cervello, condividendo con voi i miei pensieri del sogno e della realtà... fino a quando non mi prende completamente!

Questo è un linguaggio poetico, prima ancora di essere un linguaggio che utilizza le tecnologie. Damasio, riflettendo sulla rappresentazione delle percezioni delle emozioni, immagina che siano, nella mente, come finestre dalle quali il soggetto osserva. La finestra dà su un paesaggio continuamente in cambiamento, in cui figurano degli oggetti in movimento sui quali basta che cada una certa luce per renderli capaci di suggestioni; e dove suoni più o meno rumorosi attirano $o$ distolgono l'attenzione su di essi. Il nostro corpo ha un rapporto con la distribuzione nello spazio di oggetti che non sono unicamente fisici, ma possono essere argomenti, sentimenti, ragionamenti e tanti altri elementi. Per questo possiamo usare l'espressione spazi mentali, e gli spazi mentali sfuggono ai confinamenti resi necessari dalla pandemia. La possibilità che il linguaggio poetico ci offre di guardare dalla finestra si accompagna, o può accompagnarsi, con la possibilità di uscire dalla porta e di percorrere contesti, sentieri o strade.

La parola conoscenza e la parola porta in ebraico si somigliano: porta è dalet e conoscenza è da'et. Porta e conoscenza: termini che sembrano, nel nostro vocabolario e nella nostra semantica, così lontani ma che, anche per la nostra immaginazione, hanno delle strutture simboliche che si avvicinano, che li rendono uno capace di rappresentare l'altro: la porta delle conoscenze, la conoscenza come porta da aprire.

Davvero suggestivo. Quasi mi spiace riportare la conversazione alle difficoltà quotidiane, ma in questo periodo la "scuola" ha fatto i salti mortali per cercare di raggiungere tutti gli studenti e le studentesse, di inventarsi - per restare all'ultima metafora - "porte" per la conoscenza. Non sempre ci è riuscita. $E$ non si è trattato solo di disponibilità di device, 0 di connessione internet, ma di trovare il modo di motivare la presenza davanti al tablet 0 al computer, di suscitare interesse, di mantenere una qualche relazione con i nostri studenti. La difficoltà è stata enorme e, nel caso dei bambini più piccoli e dei ragazzi con disabilità, la mediazione delle famiglie è stata indispensabile. Cosa possiamo dire sul tema del rapporto fra scuola e famiglia?

La pandemia non ha creato problemi che già non fossero presenti, li ha solo disvelati. Il rapporto fra scuola e famiglia negli ultimi anni è divenuto complicato. Con le famiglie dei bambini disabili è sempre stato particolarmente delicato. Se pensiamo al corpo umano con la struttura simbolica suggerita dalle immagini di Damasio e facendo ricorso al simbolismo di De Souzenelle, penso alla figura del "conoscente" che passa la porta[6]. Il "conoscente" che passa la porta permette forse di 
capire qualcosa di ciò che può accadere a chi è genitore di un bambino o di una bambina disabile. Raffiguriamolo così: è colui che vive il paesaggio dalle finestre e non trova la porta, vive le emozioni di quello che vede negli altri e teme di non trovare le competenze per poterlo sviluppare in un percorso. La novità che è entrata nella vita delle famiglie - non più tale a dire il vero, soprattutto in tempi di pandemia - è costituita da internet. Rappresentiamo il seguente scenario: una famiglia che ha al suo interno un bambino o una bambina disabile. I genitori - come si dice "navigano" in internet e trovano quello che trovano: indicazioni, pagine suggestive, possibilità di ritenere che vi siano in qualche parte del mondo delle soluzioni ai loro problemi. Cercano e trovano, e quello che trovano non è quello che gli operatori, i tecnici dei servizi a cui si rivolgono, offrono.

Cosa può accadere? Molto schematicamente, vi sono due possibilità. Una è la negazione: i servizi negano la validità di tutto ciò che viene offerto da internet e da questo tipo di informazione; l'altra possibilità è la curiosità: gli operatori che lavorano nei servizi mostrano interesse e curiosità nei confronti di quello che quella famiglia, quei genitori, hanno scovato in internet, e chiedono di saperne di più, si informano, cercano di avere un atteggiamento di collaborazione e di curiosità nei confronti di una situazione che appare ai loro occhi molto discutibile, forse, ma nello stesso tempo non del tutto conosciuta.

Ora proviamo a inserire una variabile in questo scenario: genitori e familiari hanno avuto un contatto con un modo di realizzare la riabilitazione fortemente seducente (e ciascuno potrà mettere dietro queste parole la riabilitazione che conosce o che ha sentito ricordare come caratterizzata da questa capacità di attirare e anche di sedurre). Gli operatori dei servizi hanno già dato una valutazione negativa su quel tipo di riabilitazione. Dovrebbero quindi essere subito determinati a dare un giudizio negativo sulla scelta che stanno facendo quei genitori, quei familiari. Potrebbero però anche - ed è l'altra prospettiva - interrogarsi su quanto possa essere intervenuto a modificare quella riabilitazione dall'ultima loro valutazione. Potrebbero anche interrogarsi sulla possibilità che quella scelta riabilitativa sia accompagnata da consigli che ne permettano un adattamento e un'utilizzazione "morbida", capace, quindi, di essere compatibile con le proprie esigenze professionali di operatori.

Gustavo Zagrebelsky[7] distingue due logiche: quella dei valori, che tende all'assoluto e all'imposizione e quella dei principi che opera orientando e non imponendo, per convinzioni diffuse e non imposte. Uscendo dalla logica dell'assoluto, occorre rendere possibile un accostamento della proposta riabilitativa o educativa in termini tali da coglierne gli elementi positivi e permetterne l'adattamento nella vita reale e nel contesto in cui la vita di quel gruppo - e di quel bambino, e di quella bambina - si svolge. È la differenza fra una relazione di dominio e una relazione di cooperazione. Se ci pensiamo «anche il dominio, alla fin fine, è un genere di relazione»[8], ma disfunzionale. Divide nettamente i "gestori" dai "gestiti". In questa logica, le incognite vengono sempre attribuite a chi gestisce. Ma qualsiasi progetto, il futuro stesso, ha sempre delle incognite. L'essere gestiti comporta il non farsi carico del futuro, quindi del progetto, e dare la colpa delle incognite al gestore. È il circolo vizioso del vittimismo, che solo una logica di cooperazione può superare.

\section{La cooperazione fra i vari enti formatori è possibile solo se si instaura un vero dialogo educativo, se ci si percepisce come "comunità educante"?}

Cooperiamo perché ci sentiamo, e siamo, imperfetti: Rita Levi Montalcini ne trattò espressamente in Elogio dell'imperfezione (1990). Gli ambienti in cui svolgiamo le nostre attività sono imperfetti se non vengono ripensati in rapporto con l'originalità di chi vi svolge un'attività, vi vive un pezzetto 
della sua vita. Che non è un a-priori standardizzato. L'intreccio fra cooperazione e strutture deve tener conto dell'altro con la sua originalità e i tratti che abbiamo in comune, in una logica di comune appartenenza. $\mathrm{Ci}$ orientiamo meglio se sentiamo di appartenere a una storia comune, con la nostra originalità. Un'orchestra è composta da musicisti che suonano strumenti diversi. Non potremmo immaginare certe musiche suonate da un'orchestra composta da musicisti che suonano tutti lo stesso strumento.

Il dialogo educativo non può essere fatto senza un incontro incorporato. Raimundo Panikkar, che è stato un grande teologo e studioso delle culture, ha scritto che non esiste cultura, ideologia o religione che possa, oggi, non diciamo risolvere i problemi dell'umanità, ma parlare per il suo insieme. È necessario - sostiene Panikkar - che intervengano il dialogo e gli scambi umani che portino a una mutua fecondità. Cooperare - e l'educazione è inevitabilmente lavorare con altri - è assunzione di responsabilità.

In un saggio sul tema della responsabilità e della riflessività, Carmen Leccardi, dell'Università di Milano Bicocca, scrive che vi sono due aspetti decisivi nel rapporto fra pratiche della responsabilità e vita quotidiana:

a) le pratiche di responsabilità hanno a che fare con altri concreti, dunque sono intessute di tempi e spazi vissuti, di conflitti, di ragioni, di sentimenti ed emozioni intrecciati. Sono dimensioni dell'esperienza;

b) queste pratiche sono incorporate. La responsabilità come risposta (a sé stessi e ad altri concreti) non può essere pensata fuori dalla dimensione corporea[9].

Queste considerazioni sono preziose e ci rimandano col pensiero a una scuola finalmente in presenza, in cui anche la dimensione corporea riprenda - forse con maggior consapevolezza di prima - il proprio posto. Ne faremo certamente tesoro. Per chiudere vorrei però qualche consiglio utile anche nel caso si dovesse, nel corso del prossimo anno scolastico, ricorrere di nuovo alla Didattica a distanza. Con quali riflessioni possiamo salutarci?

Un nostro compito in tempo di pandemia può essere quello di evitare di dipendere totalmente dalle tecnologie, attribuendo alle parole non unicamente il significato che le tecnologie sembrano imporre. Uscire dalla tirannia del gergo. Come ad esempio per l'espressione "fare rete". Sottraiamola alla connessione on line e diamole un significato che impegni culturalmente la nostra partecipazione attiva. Il concetto di "rete" è oggi ampiamente usato e con varie accezioni; il concetto di "rete sociale" viene utilizzato nel 1954 dallo studioso Barnes riferendosi ai legami esistenti in una piccola parrocchia di un'isola norvegese, caratterizzati da relazioni che non erano identificabili né con quelle di lavoro, né con quelle di vicinato. Il tratto caratteristico di questa rete era dato dal fatto che appariva svincolata da spiegazioni del tipo status/ruolo e norme/valori. Vengono riconosciute come caratteristiche fondamentali e comuni a tutte le reti sociali la proprietà di produrre sostegno nei confronti dei singoli che ne fanno parte e la reciprocità degli scambi che avvengono. Una rete produttiva ha diversi attori, ciascuno dei quali sa che il proprio avvenire e la propria fortuna sono in buona parte basate sul fatto di costituire, con altri attori diversi da sé, un insieme sufficientemente stabile e nello stesso tempo flessibile e modulabile. I nodi della rete, fondamentali per la sua esistenza, sono costituiti da competenze complementari. La qualità è frutto della rete, della sua solidità, che vuol dire anche capacità di assorbire gli imprevisti. E capacità di includere e integrare elementi all'apparenza non validi. Intendere la solidità come rigidità è l'errore che viene fatto molte volte. Lo capiamo meglio facendo riferimento ad una rete che potrebbe essere presa a modello: la rete neurale. È costituita da neuroni, che possono essere anche considerati 
"nodi", connessi nel sistema nervoso periferico o in quello centrale. Hanno una continua alimentazione da stimoli esterni, che portano la rete neurale stessa ad attivarsi, riorganizzarsi elaborando continuamente nuove informazioni. Ogni neurone riceve segnali da altri neuroni grazie alle sinapsi, che permettono di trasformare le energie conflittuali in forza positiva e propositiva. Proponiamoci di fare rete:

- Non per mettere insieme, in un unico contenitore, tutte le pene. Ma per tenerle separate, ciascuna per conto suo. Se noi buttiamo in un unico mucchio le nostre paure, rischiamo di oscurare il sole. E c'è un altro rischio: ogni paura vuole essere protagonista: le paure litigano per contendersi il primato. La rissa si allarga, occupa tutto lo spazio.

- Non per trovare il o la colpevole ma il punto d'appoggio che permetta di fare un passo avanti. La ricerca del colpevole è un dispendio sterile di energie. «Che cos'è una crisi? Per rispondere a questa domanda, un buon punto di partenza potrebbe essere l'origine etimologica della parola. $<$ Crisi $>$ deriva dal sostantivo greco krisis $s_{\overline{2}}(\kappa \rho i ́ \sigma ı)_{\overline{2}}$ e dal verbo

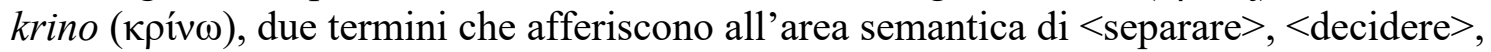
$<$ distinguere $>$. Quindi potremmo pensare alla crisi come a un momento di verità, un punto di svolta in cui la differenza tra la realtà che precede quel momento e la realtà che lo segue è molto più marcata che nella maggior parte degli altri momenti»[10].

- Perché un ostacolo diventi un punto d'appoggio.

- Per fare periscopio, guardare oltre, ampliare l'orizzonte.

- Per non cercare complicità da congiurati ma collaborazioni progettuali che valorizzino $\mathrm{i}$ ruoli istituzionali.

- Per cercare insieme depositi di energia.

- Per non lasciare indietro nessuno.

- Perché anche la persona con cui non andiamo d'accordo ha una passione. Intrecciamo le passioni e faremo la rete.

- Perché chi porta un problema può portare una risorsa.

- Perché le risorse non sono mie, o nostre. Sono dell'istituzione, e così si valorizzano.

- Per produrre non rotture. Evoluzioni.

- Perché, se hai un'idea, condividila. La sua realizzazione non sarà tua. Sarà più robusta: sarà nostra.

\section{Indicazioni bibliografiche}

- A. V. Banerjee, E. Dulfo, L'economia dei poveri. Capire la vera natura della povertà per combatterla, Feltrinelli, Milano, 2012; 2010

- F. Comunello, E. Berti, Fattoria sociale. Un contesto competente di sostegno oltre la scuola, Erickson, Trento, 2013

- A. R. Damasio, L'erreur de Descartes. La raison des émotions, Paris, Ed. O. Jacob, Paris. L'edizione italiana è presso l'editore Adelphi, 1995; 1994

- A. De Souzenelle, Il simbolismo del corpo umano, Servitium, Sotto il Monte (Bergamo), 1999;1997

- J. Diamond, Crisi. Come rinascono le nazioni, Einaudi, Torino, 2019

- R. Dunbar, La scimmia pensante. Storia dell'evoluzione umana, Il Mulino, Bologna, 2009

- J. Erpenbeck, Voci del verbo andare, Sellerio, Palermo, 2016: 2015

- D. Fassin, La Raison humanitaire. Une histoire morale du temps présent, Gallimard/Seuil, Paris, 2010 
- R. Ivekovic, Autonomia dei Balcani. Saggio di psico-politica, Raffaello Cortina editore, Milano, 1999

- C. Leccardi, Limiti della modernità. Trasformazioni del mondo e della conoscenza, Carocci, Roma, 1999

- $\quad$ R. Levi Montalcini, Elogio dell'imperfezione, Garzanti, Milano, 1990

- E. Lévinas, Totalità e infinito. Saggio sull'esteriorità, Jaca Book, Milano, 1977; 1971

- A. Mitschelinch, Il feticcio urbano. La città inabitabile, istigatrice di discordia, Einaudi, Torino, 1968; 1965

- M. C. Nussbaum, Non per profitto, Il Mulino, Bologna, 2011; 2010

- R. Panikkar, La notion des droits de l'homme est-telle un concept occidental?, in «recherches», n. 13, La Découverte, Paris, 1999

- C. Pontecorvo, A. M. Ajello, C. Zucchermaglio (a cura di), I contesti sociali dell'apprendimento, LED, Milano, 1996

- M. Tomasello, Altruisti nati. Perché cooperiamo fin da piccoli. Le basi scientifiche del nostro istinto ad aiutare il prossimo, Bollati Boringhieri, Torino, 2010; 2009

- G. Zagrebelsky, Valori e diritti. Dietro ai conflitti della politica, in "la Repubblica" del 22.2.2008

Note:

[1] Stipulati tra il $1^{\circ}$ ed il 21 novembre 1995, tali accordi prendono il nome dalla base aerea di Dayton, Ohio (USA), in cui vennero sottoscritti.

[2] La Radio Télévision Libre des Mille Collines ha svolto un ruolo significativo nel genocidio ruandese dell'aprile-luglio 1994, avendo istigato all'odio razziale contro i Tutsi e avendo dato il segnale di inizio al massacro.

[3] E. Lévinas, Totalità e infinito. Saggio sull'esteriorità, Jaca Book, Milano, 1977; 1971, p.74.

[4] A. Mitschelinch, Il feticcio urbano. La città inabitabile, istigatrice di discordia, Einaudi, Torino, 1968; 1965, p. 38.

[5] R. Ivekovic, Autonomia dei Balcani. Saggio di psico-politica, Raffaello Cortina editore, Milano, 1999, p. 13.

[6] Cfr. A. De Souzenelle, Il simbolismo del corpo umano, Servitium, Sotto il Monte (Bergamo), 1999;1997, pp. 60-61.

[7] G. Zagrebelsky, Valori e diritti. Dietro ai conflitti della politica, in "la Repubblica" del 22.2.2008

[8] J. Erpenbeck, Voci del verbo andare, Sellerio, Palermo, 2016;2015, p. 152.

[9] C. Leccardi, Limiti della modernità. Trasformazioni del mondo e della conoscenza, Carocci, Roma, 1999, p. 79.

[10] J. Diamond, Crisi. Come rinascono le nazioni, Einaudi, Torino, 2019, p. XVIII- XIX. 
